\title{
Incidental Discovery of a Patent Ductus Arteriosus in Adults
}

\author{
Harvey D. Cassidy, MD, Lynsey A. Cassidy, MD, and Joseph L. Blackshear, MD
}

Patent ductus arteriosus (PDA) is an uncommon clinical finding in adult primary care patients. However, with improved survival of premature infants at risk for PDA and an increase in cases discovered incidentally on echocardiograms performed for other purposes, the incidence of PDA is rising. Thus, primary care physicians need to be alert to the clinical situations suggesting a previously undiagnosed PDA. It is estimated that the incidence of PDA is approximately $0.02 \%$ to $0.04 \%$ in term infants and slightly higher in premature infants. PDA accounts for $6 \%$ to $11 \%$ of all congenital heart defects.

Although most cases of PDA would seem to occur sporadically, multifactorial inheritance is believed to underlie many cases. These people are thought to possess a genetic predisposition acted on by an environmental trigger that occurs at an unknown but vulnerable time during the pregnancy.

The clinical spectrum of presentation of a PDA may range from a "silent" PDA, one with no clinical manifestations but which is incidentally discovered on echocardiogram for other purposes, to patients who present with congestive heart failure, pulmonary hypertension, signs of volume overload, endocarditis, atrial fibrillation, or "recurrent pneumonia." We describe 2 cases that illustrate the discovery of an asymptomatic PDA during routine physical examination of adult patients. (J Am Board Fam Med 2009;22:214-218.)

Patent ductus arteriosus (PDA) is an uncommon clinical finding in primary care adult patients. However, with improved survival of premature infants who are known to be at risk for PDA and an increase in cases discovered incidentally on echocardiograms performed for other purposes, the incidence of PDA is rising. Primary care physicians need to be alert to the clinical situations, signs, and symptoms that might suggest a previously undiagnosed PDA. It is estimated that the incidence of PDA is approximately $0.02 \%$ to $0.04 \%$ in term infants. However, depending on estimated gestational age, the incidence in preterm neonates varies from $20 \%$ to $60 \%$ on the third day of life. ${ }^{1,2}$ PDA

This article was externally peer reviewed.

Submitted 24 October 2007; revised 30 July 2008; accepted 13 August 2008.

From Department of Family Medicine (HDC), Department of Internal Medicine (LAC), and Department of Cardiology (JLB), Mayo Clinic Florida, Jacksonville, Florida.

Funding: none.

Conflict of interest: none declared.

Corresponding author: Harvey D. Cassidy, MD, Department of Family Medicine, Mayo Clinic Florida, 4500 San Pablo Road, Jacksonville, FL 32224 (E-mail: cassidy. harvey@mayo.edu). accounts for $6 \%$ to $11 \%$ of all congenital heart defects. $^{3}$

The ductus arteriosus is a vital fetal vascular structure thought to originate from the left sixth aortic arch during embryonic development that connects the main pulmonary artery to the descending aorta. The ductus diverts blood away from the high-resistance, unexpanded fetal circulation into the descending aorta and into the fetal arterial circulation. In the fetus, the ductus remains patent because of the low arterial oxygen content and prostaglandin circulation. After delivery, however, the arterial oxygen content rises, causing a decline in pulmonary vascular resistance and immediately reversing blood flow to the ductus arteriosus. The onset of ventilation, a decline in circulating prostaglandins, and increased metabolism in the pulmonary circulation secondary to pulmonary flow promotes constriction and functional closure of the ductus generally within 10 to 15 hours after delivery, with permanent structural closure occurring within 2 to 3 weeks.

Although most cases of PDA would seem to occur sporadically, multifactorial inheritance is believed to underlie many cases. These people are thought to possess a genetic predisposition 
acted on by an environmental trigger that occurs at an unknown but vulnerable time during the pregnancy. ${ }^{4}$

The clinical spectrum of presentation of a PDA may range from a "silent" PDA, one with no clinical manifestations but which is incidentally discovered on echocardiogram for other purposes, to patients who present with congestive heart failure, pulmonary hypertension, signs of volume overload, endocarditis, atrial fibrillation, or recurrent pneumonia. We describe 2 cases illustrating the discovery of an asymptomatic PDA on routine physical examination of adult patients.

\section{Patient 1}

A healthy 55-year-old white man presented to the Mayo Clinic Department of Family Medicine to establish care and for precolonoscopic evaluation for anesthesia. The product of an uncomplicated term delivery, his childhood and adolescent medical history were unremarkable. His adult medical history was significant only for mild hyperlipidemia and benign hematuria. Family history revealed no evidence of congenital heart disease. He noted excellent exercise capacity throughout his life, and during the past 10 years he had participated in a number of long-distance running marathons without consequence. A review of systems was unrevealing and, in particular, no cardiac or respiratory difficulties were noted. His physical examination revealed a grade $2 / 6$ continuous cardiac murmur with systolic attenuation heard best at the second left intercostal space radiating to just below the left mid-clavicular chest. There was no radiation of the murmur to the back. There were no thrills, heaves, rubs, or gallops noted. There was no evidence of widened pulse pressure or apical diastolic murmur, and the second heart sound seemed to be normally split. The remainder of physical and laboratory examinations were otherwise unremarkable. An echocardiogram was performed to further assess the murmur. Left ventricular chamber size and function were normal. No regional wall motion abnormalities were noted. Both atria were normal in size. We were unable to estimate the right ventricular pressure because of an incomplete tricuspid regurgitation jet. Incidentally noted was a PDA connecting the aortic arch to the pulmonary artery (see Figure 1). Computed tomographic angiography was performed and revealed an oval-shaped
PDA of a $1.25-\mathrm{cm} \times 0.8-\mathrm{cm}$ cross-section at its midpoint. Because this seemed to be a "silent" PDA in an asymptomatic patient with no left ventricular hypertrophy or volume overload, the decision was made to continue careful follow up on a 6-month basis. Based on most recent recommendations for subacute bacterial endocarditis prophylaxis, ${ }^{5}$ no prophylaxis was recommended. However, maintenance of good oral hygiene and routine prophylactic dental care was stressed to the patient. The patient continues to participate in his routine regular exercise program to maintain cardiovascular fitness and will be followed closely in the family medicine clinic.

\section{Patient 2}

A 73-year-old white woman presented to the Mayo Clinic Division of Allergy and Immunology requesting evaluation for a 10-year history of chronic cough, which she felt was caused by her "allergies." She provided a history of "more than the usual amount" of bronchial problems throughout her life, but denied a history of asthma or chronic pulmonary conditions and was a life-long nonsmoker. She was the product of a term pregnancy with an uneventful neonatal period. She denied any history of murmur or cardiorespiratory problems as a child. However, 10 years before this appointment she had been advised that she had a "slight murmur." No further evaluation was performed at that time. One year before her presentation at Mayo Clinic she had an echocardiogram performed for an unknown reason which, by her report, showed "something wrong with blood flowing out of my heart." She stated that her physicians were not unduly concerned about this and she was given no additional recommendations at that time.

Her medical history included a hospitalization for pneumonia 4 years prior and, most recently, 1 month before her presentation at our allergy clinic she was evaluated at a nearby emergency department for "pneumonia" and was treated with a 10 day course of antibiotics.

During a review of systems she noted atypical chest discomfort that did not seem to be cardiac in nature. She denied any paroxysmal nocturnal dyspnea or orthopnea, and there was no history of any functional limitations in her routine activities.

During physical examination the cardiac examination was notable for a prominent grade $3 / 6$ 


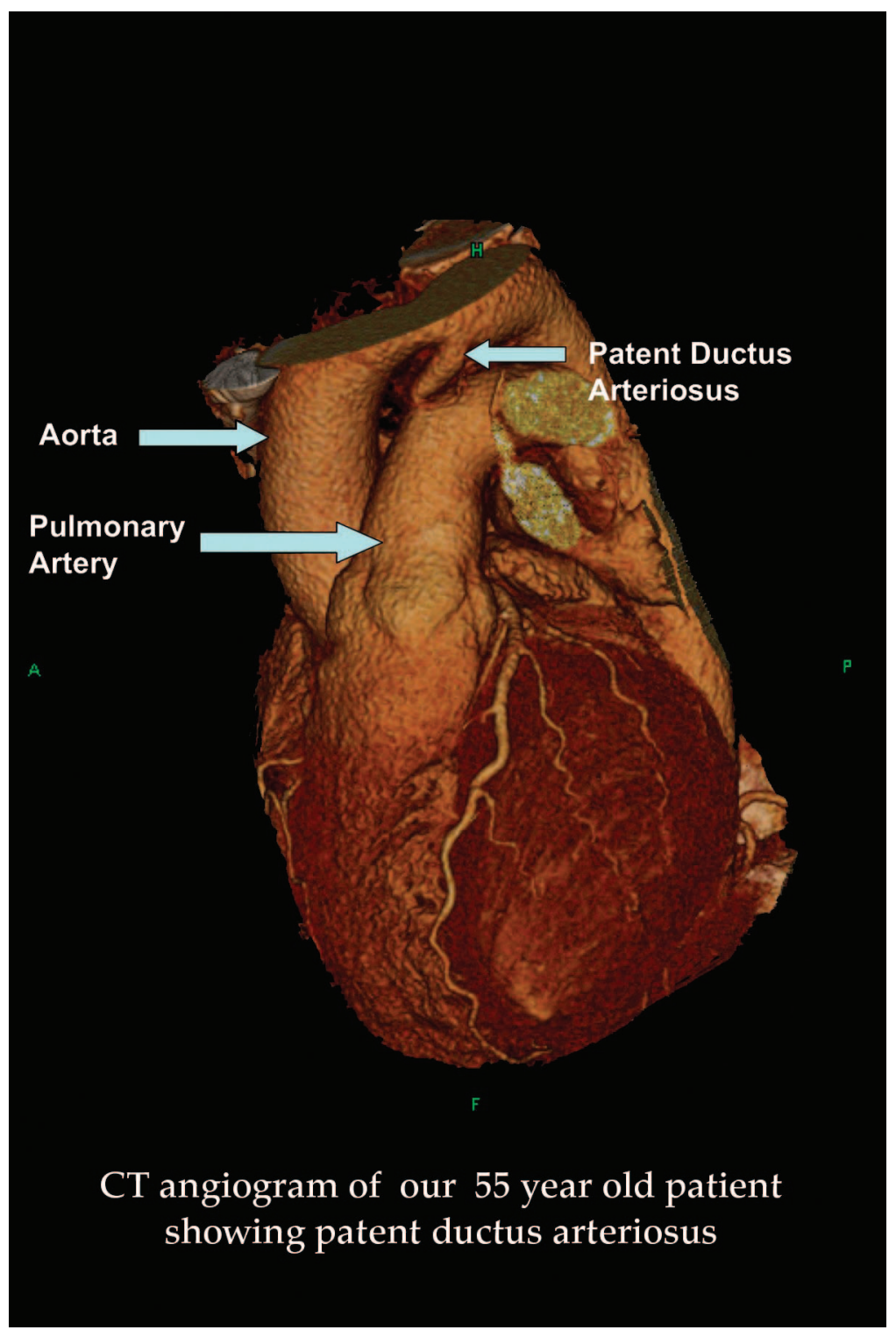

Figure 1. Computed tomographic angiogram of our 55-year-old patient showing patent ductus arteriosus.

systolic murmur best heard at the left upper sternal border second intercostal space with radiation into the anterior left chest. No gallops, thrills, heaves, or rubs were noted. The second heart sound was physiologically split, suggesting an absence of significant pulmonary hypertension. Peripheral pulses seemed to be normal, with no evidence of pulse deficit. No diastolic murmurs were heard. As part of her evaluation, an exercise echocardiogram was performed and revealed no regional wall motion abnormalities and no evidence of ischemia. Resting echocardiogram revealed the presence of a PDA in the setting of normal left ventricular function. Doppler derived pulmonary artery mean pressure was $26 \mathrm{~mm} \mathrm{Hg}$, consistent with mild pulmonary hypertension (25-35 mm Hg). Moderate left and right atrial enlargement and a mildly enlarged main pulmonary artery were noted.

Cardiology consultation was requested and, in light of her mild pulmonary hypertension, dilated pulmonary artery, left atrial enlargement, and recurrent pneumonia, the option of transcatheter or surgical closure was discussed with the patient. However, because this patient had no loss of functional capacity and preferred noninvasive therapy, she chose a conservative approach with a follow-up echocardiogram in 1 year unless she should become 
symptomatic or have a decline in functional capacity. At this time, the patient continues to do well and has no functional limitations. Maintenance of good oral hygiene and appropriate routine dental care was stressed to the patient.

\section{Discussion}

The above cases illustrate previously undiagnosed PDA in adult patients. Although the incidence of PDA is highest in preterm infants, both of these cases involved adults who were term infants at birth with an unremarkable neonatal course. However, the incidence of PDA has increased over the years because advances in medical science have improved the survival of premature infants.

Clinical manifestations of PDA may vary greatly among people and are dependent on size of the ductus, the age of the patient, the pressure differential across the ductus, and the presence or absence of pulmonary hypertension. Some patients with an underlying PDA may be quite symptomatic, presenting with congestive heart failure, pulmonary hypertension, signs of volume overload, atrial fibrillation, recurrent pneumonia, or other complications known to be associated with PDA. Others have no signs or symptoms, and a "silent" PDA may be discovered only incidentally on an echocardiogram done for other purposes. Clinical recognition of a PDA in an adult who was born premature is usually made when a murmur is present or when oxygen requirement increases during the course of recovery from lung disease. ${ }^{6}$ Asymptomatic PDAs, tolerated for many years without clinical signs or symptoms, may become clinically significant in patients with unrepaired PDAs when acquired conditions such as recurrent pneumonia, the development of chronic obstructive pulmonary disease, or the manifestations of valvular or ischemic heart disease are superimposed.

Physical examination findings can vary significantly depending on the pressure dynamics across the ductus. Findings, when present, may include a wide pulse pressure with prominent or bounding peripheral pulses, hyperdynamic apical pulse, continuous murmur localized to the left infraclavicular area or upper left sternal border, diastolic rumble, narrow or single S2 suggestive of increased pulmonary vascular resistance, visible carotid pulsations, and suprasternal notch thrill. Chest radiographs in patients with PDA may be normal or may show increased pulmonary vascular markings, enlargement of the main pulmonary artery, and occasionally calcification of the ductus, particularly evidenced in adults.

Typically, echocardiogram is used to confirm the diagnosis of PDA as well as to determine characteristics such as size, shape, and location of the persistent vascular channel. It also may provide very useful information about the effect of the PDA on cardiovascular dynamics such as pulmonary artery pressure and atrial size, as well as left ventricular size and function. Magnetic resonance imaging and computed tomographic angiography may provide further useful information for characterizing the PDA as well as establishing the surgical candidacy of the patient. Cardiac catheterization may be useful to determine that the patient is hemodynamically an appropriate candidate for closure.

Treatment of PDA in premature infants is controversial. According to Clyman and Chorne, "in recent years there has been a growing debate about whether or not to treat a persistent PDA during the neonatal period. Preterm infants have a high rate of spontaneous PDA closure during the first 2 years." They suggest that the early treatment may unnecessarily expose infants to both pharmacological and surgical treatment that they may not need and which could produce unnecessary morbidity or mortality. Recently, in preterm infants, conservative treatments using the adjustment of ventilation by reducing inspiratory time and giving more positive end-expiratory pressure and fluid restriction exceeding $130 \mathrm{~mL} / \mathrm{kg} /$ day beyond day 3 has produced a high closure rate of PDA in at least one study. ${ }^{2}$ In preterm infants, pharmacological or transcatheter occlusion are the preferred alternatives to surgical ligation in most cases. ${ }^{8}$ In the older infant or child, transcatheter occlusion is generally the preferred treatment of choice. ${ }^{9}$ For adults, transcatheter occlusion of the patent ductus is the preferred treatment when possible. ${ }^{8}$ However, in adults with large, unfavorably shaped ducts, surgical ligation is a safe and effective alternative although calcification of the ductus may increase the technical difficulty of the procedure.

Small PDAs (those with ductal diameter of 1.5$2.5 \mathrm{~mm}$ ) without hemodynamic overload are generally closed because of the risk of subacute bacterial endocarditis. Medium and large-sized PDAs (larger than $2.5 \mathrm{~mm}$ ) should be closed to prevent 
volume overload of the left ventricle, prevent pulmonary vascular obstructive disease, and to treat congestive heart failure. ${ }^{3}$ Closure of "silent" PDAs remains controversial and requires further research.

The need for bacterial endocarditis prophylaxis in an unrepaired PDA remains controversial. Subacute bacterial endocarditis has been reported in both symptomatic and "silent" PDAs. Recent guidelines from the American Heart Association of the Committee on Rheumatic Fever, Endocarditis and Kawasaki disease do not recommend routine subacute bacterial endocarditis prophylaxis for unrepaired PDAs. However, decisions regarding prophylaxis in patients should be made in conjunction with a cardiologist who is well-versed in the evaluation and management of congenital heart disease. Meticulous attention to oral hygiene and regular access to appropriate dental care should be emphasized to all patients. ${ }^{5}$

\section{Conclusion}

The incidence of PDA is rising, and it seems likely that we will continue to discover previously unrecognized cases of PDA in our adult patient populations. Appropriate evaluation of these patients by primary care physicians and our cardiology colleagues is vital in determining appropriate therapy. Newer percutaneous transcatheter occlusive devices have been shown to be very safe and effective in both children and adults, making the need for surgical ligation less common. For those asymptomatic patients without complications related to persistence of the ductus, close follow-up is necessary to monitor for development of symptoms and sequelae of progressive disease. These patients should and can be managed in conjunction with our cardiology colleagues who are well-versed in the evaluation and management of congenital heart disease. Asymptomatic patients may not require closure of the PDA, but this can only be determined after a thorough evaluation has been completed.

\section{References}

1. Doyle T, Kahanauph A, Graham T Jr. Overview of PDA. Up to date online, version 16.3, last updated June 5, 2008.

2. Vanhaesebrouck S, Zonnenberg I, Vandervoort P, Bruneel E, Van Hoestenberghe MR, Theyskens C. Conservative treatment for patent ductus arteriosus in the preterm. Arch Dis Child Fetal Neonatal Ed 2007;92:F244-7.

3. Rao PS. Percutaneous closure of patent ductus arteriosus: state of the art. J Invasive Cardiol 2007;19: 299-302.

4. Schneider DJ, Moore JW. Patent ductus arteriosus. Circulation 2006;114:1873-82.

5. Wilson W, Taubert KA, Gewitz M, et al. Prevention of infective endocarditis: guidelines from the American Heart Association: a guideline from the American Heart Association Rheumatic Fever, Endocarditis, and Kawasaki Disease Committee, Council on Cardiovascular Disease in the Young, and the Council on Clinical Cardiology, Council on Cardiovascular Surgery and Anesthesia, and the Quality of Care and Outcomes Research Interdisciplinary Working Group. Circulation. 2007;116:1736-54.

6. Finberg L. Saunders Manual of Pediatric Practice. St. Louis (MO): WB Saunders Co; 1998:559-61.

7. Clyman RI, Chorne N. Patent ductus arteriosus: evidence for and against treatment. J Pediatrics 2007; 150:216-9.

8. Atiq M, Aslam N, Kazmi K. Transcatheter closure of small-to-large patent ductus arteriosus with different devices: queries and challenges. J Invasive Cardiol 2007;19:295-8.

9. Alario AJ, Birnkrant JD. Practical guide to the care of the pediatric patient: Practical Guide Series, 2nd ed. Philadelphia (PA):Mosby; 2007:387-8. 